\title{
Bianchi Type-I Universe with Cosmological Constant and Quadratic Equation of State in $f(R, T)$ Modified Gravity
}

\author{
G. P. Singh and Binaya K. Bishi \\ Department of Mathematics, Visvesvaraya National Institute of Technology, Nagpur 440010, India \\ Correspondence should be addressed to Binaya K. Bishi; binaybc@gmail.com
}

Received 14 August 2015; Revised 2 November 2015; Accepted 8 November 2015

Academic Editor: Enrico Lunghi

Copyright (C) 2015 G. P. Singh and B. K. Bishi. This is an open access article distributed under the Creative Commons Attribution License, which permits unrestricted use, distribution, and reproduction in any medium, provided the original work is properly cited. The publication of this article was funded by SCOAP ${ }^{3}$.

\begin{abstract}
This paper deals with the study of Bianchi type-I universe in the context of $f(R, T)$ gravity. Einstein's field equations in $f(R, T)$ gravity have been solved in the presence of cosmological constant $\Lambda$ and quadratic equation of state (EoS) $p=\alpha \rho^{2}-\rho$, where $\alpha \neq 0$ is a constant. Here, we have discussed two classes of $f(R, T)$ gravity; that is, $f(R, T)=R+2 f(T)$ and $f(R, T)=f_{1}(R)+f_{2}(T)$. A set of models has been taken into consideration based on the plausible relation. Also, we have studied some physical and kinematical properties of the models.
\end{abstract}

\section{Introduction}

It is known that [1-5] in the present scenario, our universe is accelerating. However, final satisfactory explanation about physical mechanism and driving force of accelerated expansion of the universe is yet to achieve as human mind has not achieved perfection. From the modern cosmology, it is known that a point of universe is filled with dark energy. It has been addressed by various slow rolling scalar fields. It is supposed that the dark energy is responsible for producing sufficient acceleration in the late time of evolution of the universe. Thus, it is much more essential to study the fundamental nature of the dark energy and several approaches have been made to understand it. The cosmological constant is assumed to be the simplest candidate of dark energy. It is the classical correction made to Einstein's field equation by adding cosmological constant to the field equations. The introduction of cosmological constant to Einstein's field equation is the most efficient way of generating accelerated expansion, but it faces serious problems like finetuning and cosmic coincidence problem in cosmology $[6,7]$. Quintessence [8], phantom [9], k-essence [10], tachyons [11], and Chaplygin gas [12] are the other representative of dark energy. However, there is no direct detection of such exotic fluids. Researchers are taking an interest in exploring dark energy due to the lack of strong evidence of existence of dark energy. Several authors (Pimentel and Diaz-Rivera [13], Singh et al. [14], Singh et al. [15], and Jamil and Debnath [16]) have discussed cosmological model with cosmological constant in different contexts.

Dark energy can be explored in several ways, and modifying the geometric part of the Einstein-Hilbert action [17] is treated as the most efficient possible way. Based on its modifications, several alternative theories of gravity came into existence. Some of the modified theories of gravity are $f(T), f(R), f(G)$, and $f(R, T)$ gravity. These models are proposed to explore the dark energy and other cosmological problems. Sharif and Azeem [18] discussed the Cosmological evolution for dark energy models in $f(T)$ gravity. Jamil et al. [19] have studied the stability of the interactive models of the dark energy, matter, and radiation for a FRW model in $f(T)$ gravity. Generalized second law of thermodynamics in $f(T)$ gravity with entropy corrections has been studied by Bamba et al. [20]. In this work, they have used the power law and logarithmic corrected form of entropy for cosmological horizon and analysed the validity of the generalized second law of thermodynamics in specific scenarios of the quintessence and the phantom energy dominated eras. The $f(R)$ modified 
theory produces both cosmic inflation and mimic behavior of dark energy, including present cosmic acceleration [21-23]. Amendola et al. [24] have discussed the cosmologically viable conditions in $f(R)$ theory, which describe the dark energy models. Jamil et al. [25] have analysed the $f(R)$ tachyon cosmology by the Noether symmetry approach. Azadi et al. [26] have discussed the static cylindrically symmetric vacuum solutions in Weyl coordinates in the context of the metric $f(R)$ theories of gravity. This article is devoted to construct the family of solutions with constant Ricci scalar $\left(R=R_{0}\right)$ explicitly and its possible relation to the Linet-Tian solution in general relativity. Momeni and Gholizade [27] discussed the constant curvature solutions in cylindrically symmetric metric $f(R)$ gravity. In this paper, they have proved that, in $f(R)$ gravity, the constant curvature solution in cylindrically symmetric cases is only one member of the most generalized Tian family in general relativity and further shown that constant curvature exact solution is applicable to the exterior of a string.

The basic paper on $f(R, T)$ modified gravity was investigated by Harko et al. [28]. From the literature, it is found that Barrientos and Rubilar have pointed out that Harko et al. have missed an essential term, which has consequences in the equation of motion of test particles. Thus, the corrected derivation of this equation of motion is presented by Barrientos and Rubilar [29], who also discussed some of its consequences. Jamil et al. [30] have studied the reconstruction of some cosmological models in $f(R, T)$ gravity, in which they have shown that dust fluid reproduces $\Lambda C D M$, phantom-non-phantom era and the phantom cosmology. Jamil et al. [31] have proved that the first law of black hole thermodynamics is violated for $f(R, T)$ gravity in general, but there might be some special case exit in which the first law of black hole thermodynamics is recovered. Momeni et al. [32] have investigated Noether symmetry issue for nonminimally $f(R, T)$ model and mimetic $f(R)$. They have pointed out that Noether symmetry is able to provide a very excellent way to study cosmological implications of extended $f(R)$ theories. We have observed from the literature that Bianchi typeI model is one of the important anisotropic cosmological models and hence it is widely studied in general relativity and alternative theories of gravitation. The Bianchi type-I model is discussed by Jamil et al. $[33,34]$ in different contexts. Recently, authors like Sahoo and Sivakumar [35], Ahmed and Pradhan [36], and Pradhan et al. [37] have investigated the cosmological models with cosmological constant in $f(R, T)$ gravity for different Bianchi type space-time.

Quadratic equation of state is needed to explore in cosmological models due to its importance in brane world model and the study of dark energy and general relativistic dynamics for different models. The general form of the quadratic equation of state is given by

$$
p=p_{0}+\alpha \rho+\beta \rho^{2}
$$

where $p_{0}, \alpha$, and $\beta$ are parameters. Equation (1) is nothing but the first term of Taylor expansion of any equation of state of the form $p=p(\rho)$ about $\rho=0$.

Nojiri and Odintsov [38] have studied the final state and thermodynamics of a dark energy universe, in which they discussed the model by considering the equation state of the form $p=f(\rho)$. Ananda and Bruni discussed the cosmological models by considering different form of nonlinear quadratic equation of state. Ananda and Bruni [39] have investigated the general relativistic dynamics of RW models with a nonlinear quadratic equation of state and analysed that the behaviour of the anisotropy at the singularity found in the brane scenario can be recreated in the general relativistic context by considering an equation of state of form (1). Also they have discussed the anisotropic homogeneous and inhomogeneous cosmological models in general relativity with the equation of state of the form

$$
p=\alpha \rho+\frac{\rho^{2}}{\rho_{c}},
$$

and they tried to isotropize the universe at early times when the initial singularity is approached. Astashenok et al. [40] have analysed phantom cosmology without big rip singularity, in which they have considered the equation of state of the form $p=-\rho-f(\rho)$. In our present study, we have considered the quadratic equation of state of the form

$$
p=\alpha \rho^{2}-\rho
$$

where $\alpha \neq 0$ is a constant quantity and such type of consideration does not affect the quadratic nature of equation of state.

Nojiri and Odintsov [41] studied the effect of modification of general equation of state of dark energy ideal fluid by the insertion of inhomogeneous, Hubble parameter dependent term in the late-time universe. The quadratic equation of state may describe the dark energy or unified dark energy [41, 42]. Rahaman et al. [43] investigated the construction of an electromagnetic mass model using quadratic equation of state in the context of general theory of relativity. Feroze and Siddiqui [44] studied the general situation of a compact relativistic body by taking a quadratic equation of state for the matter distribution. Maharaj and Mafa Takisa [45] have investigated the regular models with quadratic equation of state. They have considered static and spherically symmetric space-time with a charged matter distribution and found new exact solutions to the Einstein-Maxwell system of equations which are physically reasonable.

A cosmological model based on a quadratic equation of state unifying vacuum energy, radiation, and dark energy has been discussed by Chavanis [46] and also a cosmological model describing the early inflation, the intermediate decelerating expansion, and the late accelerating expansion by a quadratic equation of state has been investigated by the same author [47]. Strange quark star model with quadratic equation of state has been investigated by Malaver [48] and they have obtained a class of models with quadratic equation of state for the radial pressure that correspond to anisotropic compact sphere, where the gravitational potential $Z$ depends on an adjustable parameter $n$. Recently, Reddy et al. [49] have studied the Bianchi type-I cosmological model with quadratic equation of state in the context of general theory of relativity.

Motivated by the aforesaid research, we have investigated the Bianchi type-I cosmological model in $f(R, T)$ gravity with 
quadratic equation of state and cosmological constant. Here, we have discussed two classes of $f(R, T)$ gravity.

\section{Gravitational Field Equations of $f(R, T)$ Modified Gravity Theory}

Let us consider the action for the modified gravity as

$$
S=\int\left(\frac{f(R, T)}{16 \pi G}+L_{m}\right) \sqrt{-g} d^{4} x
$$

where $f(R, T)$ is the arbitrary function of $R$ and T. $R$ is the Ricci scalar and $T$ is the trace of the stress energy tensor of the matter $T_{i j} . L_{m}$ is the matter Lagrangian density. For the choice of $f(R, T)$, we will get the action for the different theories. If $f(R, T) \equiv f(R)$ and $f(R, T) \equiv R$, then (4) represents the action for $f(R)$ gravity and general relativity, respectively. The stress energy tensor of matter is defined as

$$
T_{i j}=-\frac{2}{\sqrt{-g}} \frac{\delta\left(\sqrt{-g} L_{m}\right)}{\delta g^{i j}},
$$

and its stress by $T=g^{i j} T_{i j}$. If we consider that the matter Lagrangian density $L_{m}$ of matter depends only on $g_{i j}$ and not on its derivatives, then it will lead us to

$$
T_{i j}=g_{i j} L_{m}-2 \frac{\partial L_{m}}{\partial g^{i j}} .
$$

By varying action (4) with respect to the metric tensor component $g_{i j}$, we have

$$
\begin{aligned}
& f_{R}(R, T) R_{i j}-\frac{1}{2} f(R, T) g_{i j}+\left(g_{i j} \square-\nabla_{i} \nabla_{j}\right) f_{R}(R, T) \\
& \quad=\left(8 \pi-f_{T}(R, T)\right) T_{i j}-f_{T}(R, T) \Theta_{i j},
\end{aligned}
$$

where

$$
\Theta_{i j}=-2 T_{i j}+g_{i j} L_{m}-2 g^{l k} \frac{\partial^{2} L_{m}}{\partial g^{i j} \partial g^{l k}} .
$$

Here, $f_{T}(R, T)=\partial f(R, T) / \partial T, f_{R}(R, T)=\partial f(R, T) / \partial R$, $\square \equiv \nabla^{i} \nabla_{i}$ is the De Alembert's operator, and $T_{i j}$ is the standard matter energy momentum tensor derived from the Lagrangian $L_{m}$. By contracting (7), we obtained the relation between $R$ and $T$ as

$$
\begin{array}{r}
f_{R}(R, T) R+3 \square f_{R}(R, T)-2 f(R, T) \\
\quad=8 \pi T-f_{T}(R, T) T-f_{T}(R, T) \Theta,
\end{array}
$$

where $\Theta=g^{i j} \Theta_{i j}$. From (7) and (9), the gravitational field equations can be written as

$$
\begin{aligned}
f_{R}(R, T)\left(R_{i j}-\frac{1}{3} R g_{i j}\right)+\frac{1}{6} f(R, T) g_{i j} \\
=\left(8 \pi-f_{T}(R, T)\right)\left(T_{i j}-\frac{1}{3} T g_{i j}\right) \\
\quad-f_{T}(R, T)\left(\Theta_{i j}-\frac{1}{3} \Theta g_{i j}\right)+\nabla_{i} \nabla_{j} f_{R}(R, T) .
\end{aligned}
$$

The perfect fluid form of the stress energy tensor of the matter Lagrangian is given by

$$
T_{i j}=(\rho+p) u_{i} u_{j}-p g_{i j}
$$

where $u^{i}=(1,0,0,0)$ is the four-velocity vector and satisfies the relation $u^{i} u_{i}=1$ and $u^{i} \nabla_{j} u_{i}=0 . \rho$ and $p$ are the energy density and pressure of the fluid, respectively. From (8), we have

$$
\Theta_{i j}=-2 T_{i j}-p g_{i j}
$$

It is to note that the functional $f(R, T)$ depends on the physical nature of the matter field through tensor $\Theta_{i j}$. Thus, each choice of $f(R, T)$ leads us to different cosmological models. Harko et al. [28] presented three classes of $f(R, T)$ as follows:

$$
f(R, T)=\left\{\begin{array}{l}
R+2 f(T) \\
f_{1}(R)+f_{2}(T) \\
f_{1}(R)+f_{2}(R) f_{3}(T) .
\end{array}\right.
$$

In this present work, we have discussed two classes of $f(R, T)$; that is, $f(R, T)=R+2 f(T)$ and $f(R, T)=f_{1}(R)+f_{2}(T)$.

For the choice of $f(R, T)=R+2 f(T)$ and with the help of (11) and (12), (7) takes the form

$$
G_{i j}=\left(8 \pi+2 f^{\prime}(T)\right) T_{i j}+\left(2 p f^{\prime}(T)+f(T)\right) g_{i j},
$$

which is the gravitational field equation in $f(R, T)$ modified gravity for the class $f(R, T)=R+2 f(T)$. For the choice of $f(R, T)=f_{1}(R)+f_{2}(T)$ and with the help of (11) and (12), (7) takes the form

$$
\begin{aligned}
& f_{1}^{\prime}(R) R_{i j}-\frac{1}{2} f_{1}(R) g_{i j}+\left(g_{i j} \square-\nabla_{i} \nabla_{j}\right) f_{1}^{\prime}(R) \\
& =\left(8 \pi+f_{2}^{\prime}(T)\right) T_{i j}+\left(f_{2}^{\prime}(T) p+\frac{1}{2} f_{2}(T)\right) g_{i j}
\end{aligned}
$$

which is regarded as the gravitational field equation in $f(R, T)$ modified gravity for the class $f(R, T)=f_{1}(R)+f_{2}(T)$.

\section{Field Equations and Cosmological Model for $f(R, T)=R+2 \boldsymbol{f}(T)$}

In $f(R, T)$ theory, the gravitational field equation (14) in the presence of cosmological constant $\Lambda$ is given as

$$
G_{i j}=\left[8 \pi+2 f^{\prime}(T)\right] T_{i j}+\left[2 p f^{\prime}(T)+f(T)+\Lambda\right] g_{i j},
$$


where prime denotes differentiation with respect to the argument. For the choice of $f(T)=\lambda T,(16)$ takes the form

$$
G_{i j}=[8 \pi+2 \lambda] T_{i j}+[\lambda \rho-p \lambda+\Lambda] g_{i j}
$$

Let us consider the Bianchi type-I space-time in the form

$$
d s^{2}=d t^{2}-X_{1}^{2} d x^{2}-X_{2}^{2} d y^{2}-X_{3}^{2} d z^{2}
$$

where $X_{1}, X_{2}$, and $X_{3}$ are function of $t$ only. The field equation (17) for the line element (18) takes the form

$$
\begin{array}{r}
\frac{\dot{X}_{1} \dot{X}_{2}}{X_{1} X_{2}}+\frac{\dot{X}_{1} \dot{X}_{3}}{X_{1} X_{3}}+\frac{\dot{X}_{2} \dot{X}_{3}}{X_{2} X_{3}}=-(8 \pi+3 \lambda) \rho+p \lambda-\Lambda \\
\frac{\ddot{X}_{2}}{X_{2}}+\frac{\ddot{X}_{3}}{X_{3}}+\frac{\dot{X}_{2} \dot{X}_{3}}{X_{2} X_{3}}=(8 \pi+3 \lambda) p-\lambda \rho-\Lambda \\
\frac{\ddot{X}_{1}}{X_{1}}+\frac{\ddot{X}_{3}}{X_{3}}+\frac{\dot{X}_{1} \dot{X}_{3}}{X_{1} X_{3}}=(8 \pi+3 \lambda) p-\lambda \rho-\Lambda \\
\frac{\ddot{X}_{1}}{X_{1}}+\frac{\ddot{X}_{2}}{X_{2}}+\frac{\dot{X}_{1} \dot{X}_{2}}{X_{1} X_{2}}=(8 \pi+3 \lambda) p-\lambda \rho-\Lambda .
\end{array}
$$

\section{Solution Procedure}

Now, our problem is to solve Einstein's modified field equations (19)-(22). Here, the system has four equations and six unknowns $\left(X_{1}, X_{2}, X_{3}, p, \rho\right.$, and $\left.\Lambda\right)$. To obtain the complete solution, we need two more physically plausible relations. The considered two physically plausible relations are

(1) quadratic equation of state;

(2) expansion law:

(a) power law:

$$
V=V_{0} t^{3 n}
$$

(b) exponential law:

$$
V=V_{0} e^{\beta t}
$$

where $n$ and $\beta$ are the positive constant quantity. According to the choice of expansion law, we have obtained two different models of the Bianchi type-I universe.

4.1. Power Law Model. With the help of (20)-(22), we have obtained the metric potentials as

$$
X_{i}(t)=X_{i 0} V^{1 / 3} \exp \left[\int \frac{X_{0 i}}{V}\right], \quad i=1,2,3,
$$

where $X_{i 0}$ and $X_{0 i}$ are constant of integration $(i=1,2,3)$ which satisfies the relation $\prod_{i=1}^{3} X_{i 0}=1$ and $\sum_{i=1}^{3} X_{0 i}=0$. From (19)-(20) and along with (3), we have got

$$
\rho^{2}=\frac{1}{\alpha(8 \pi+2 \lambda)}\left[\frac{\ddot{X}_{2}}{X_{2}}+\frac{\ddot{X}_{3}}{X_{3}}-\frac{\dot{X}_{1} \dot{X}_{2}}{X_{1} X_{2}}-\frac{\dot{X}_{1} \dot{X}_{3}}{X_{1} X_{3}}\right] .
$$

Using (23) in (25), we have the metric potential as

$$
X_{i}(t)=X_{i 0} V^{1 / 3} \exp \left[\frac{-X_{0 i} t^{-3 n+1}}{(3 n-1) V_{0}}\right], \quad i=1,2,3 .
$$

The directional Hubble parameters are obtained as $H_{i}=$ $n / t+X_{0 i} / V_{0} t^{3 n}, i=1,2,3$. The Hubble parameter $(H)$, deceleration parameter $(q)$, expansion scalar $(\Theta)$, and Shear scalar $\left(\sigma^{2}\right)$ are as follows:

$$
\begin{aligned}
H & =\frac{n}{t}, \\
q & =-1+\frac{1}{n}, \\
\Theta & =3 \frac{n}{t}, \\
\sigma^{2} & =\frac{X_{02}^{2}+X_{03}^{2}+X_{02} X_{03}}{V_{0}^{2} t^{6 n}} .
\end{aligned}
$$

Using the observational value for $q=-0.33 \pm 0.17$ [50], we have restricted $n$ as $n \in(1.19,2)$ in case of power law model. Here, we noticed that $H, \Theta$, and $\sigma^{2}$ die out for larger values of $t$. With the help of (27) from (26), the energy density is obtained as

$$
\rho^{2}=\frac{1}{(4 \pi+\lambda) \alpha}\left[\frac{X_{02}^{2}+X_{03}^{2}+X_{02} X_{03}}{V_{0}^{2}} \frac{1}{t^{6 n}}-\frac{n}{t^{2}}\right]
$$

Using (29) in (3), we have the pressure as follows:

$$
\begin{aligned}
p= & \frac{\left(X_{02}^{2}+X_{03}^{2}+X_{02} X_{03}\right) t^{-6 n+2}-V_{0}^{2} n}{(4 \pi+\lambda) V_{0}^{2} t^{2}} \\
& -\sqrt{\frac{\left(X_{02}^{2}+X_{03}^{2}+X_{02} X_{03}\right) t^{-6 n+2}-V_{0}^{2} n}{(4 \pi+\lambda) V_{0}^{2} \alpha t^{2}}} .
\end{aligned}
$$

With the help of (27)-(30) from (19), the cosmological constant $\Lambda$ is obtained as

$$
\Lambda=\frac{-4}{(4 \pi+\lambda) V_{0}^{2} t^{6 n+2}}\left[(2 \pi+\lambda)(4 \pi+\lambda) V_{0}^{2} t^{6 n+2}\right.
$$

$$
-\sqrt{\frac{\left(X_{02}^{2}+X_{03}^{2}+X_{02} X_{03}\right) t^{-6 n+2}-V_{0}^{2} n}{(4 \pi+\lambda) V_{0}^{2} \alpha t^{2}}}
$$




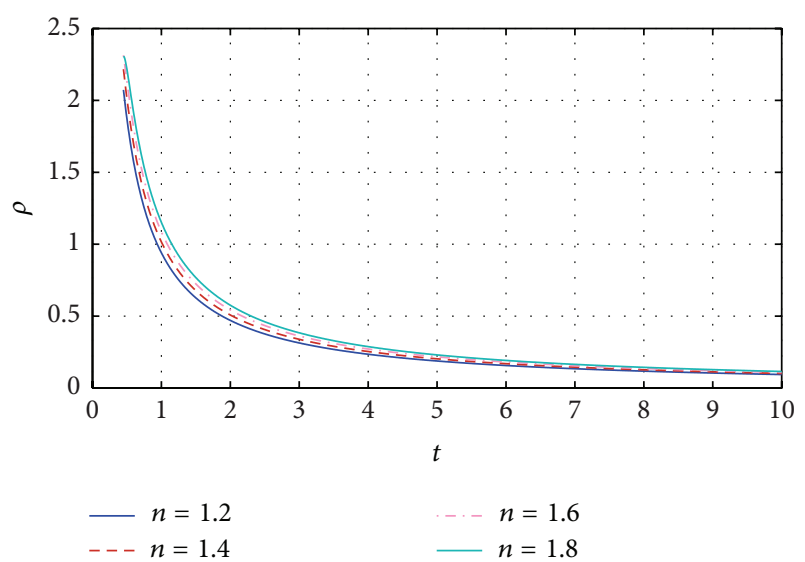

Figure 1: Variation of energy density $\rho$ against time $t$ for $\lambda=1$, $\alpha=-0.1, V_{0}=1, X_{02}=0.01, X_{03}=0.01$, and different $n(1.2,1.4,1.6,1.8)$.

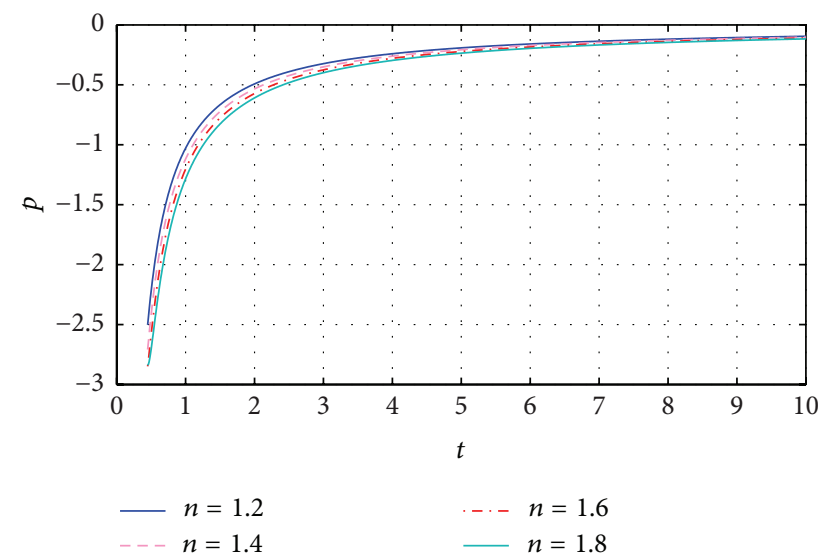

FIGURE 2: Variation of pressure $p$ against time $t$ for $\lambda=1, \alpha=-0.1$, $V_{0}=1, X_{02}=0.01, X_{03}=0.01$, and different $n(1.2,1.4,1.6,1.8)$.

$$
\begin{aligned}
& -\left(\pi+\frac{\lambda}{2}\right)\left(X_{02}^{2}+X_{03}^{2}+X_{02} X_{03}\right) t^{2} \\
& \left.+\frac{1}{4}(3 \lambda n+\lambda+12 n \pi) V_{0}^{2} n t^{6 n}\right] .
\end{aligned}
$$

Figures 1 and 2 show the variation of energy density $\rho$ and pressure $p$ against time $t$ for different values as in the figures. Here, we noticed that $\rho, p \rightarrow 0$ when $t \rightarrow \infty$. In the increase of $n$, energy density and pressure increase and decrease, respectively. Figure 3 represents the variation of cosmological constant $\Lambda$ against time $t$ for different values as in the figures. It is observed that cosmological constant $\Lambda$ is decreasing with the increase of $n$ and with the evolution of time it approaches towards zero. Variation of different energy conditions against time for different $n$ is presented in Figure 4. We observed from the figure that DEC (dominant energy

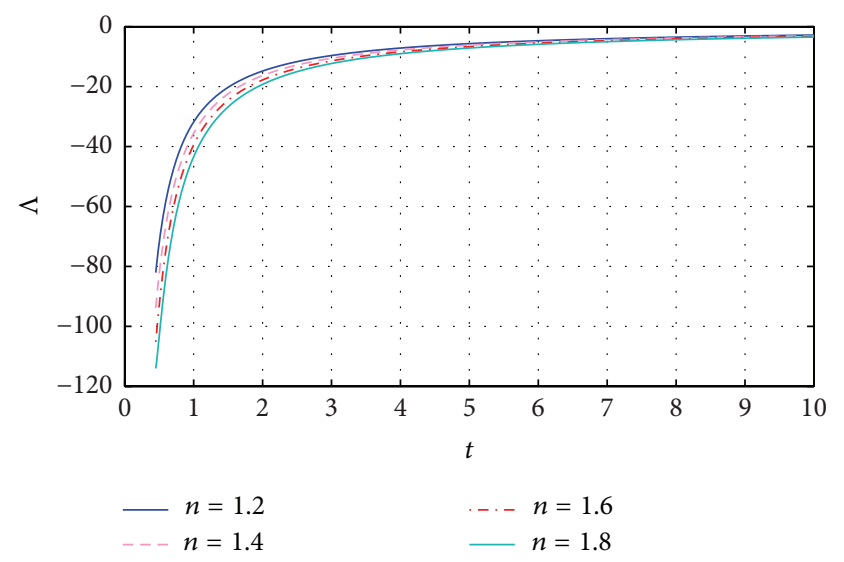

FIgURE 3: Variation of cosmological constant $\Lambda$ against time $t$ for $\lambda=1, \alpha=-0.1, V_{0}=1, X_{02}=0.01, X_{03}=0.01$, and different $n(1.2,1.4,1.6,1.8)$.

condition, $\rho-p \geq 0$ ) is satisfied, but NEC (null energy condition, $\rho+p \geq 0$ ) and SEC (strong energy condition, $\rho+3 p \geq 0$ ) are violated in this case. This violation may be responsible for the accelerated expansion of the universe.

4.2. Exponential Law Model. In this case, with the help of (24) in (25), we have found the metric potential as

$$
\begin{array}{r}
X_{i}(t)=X_{i 0} V^{1 / 3} \exp \left[-\left(\frac{-\beta^{2} V_{0} t+3 X_{0 i} e^{-\beta t}}{3 \beta V_{0}}\right)\right], \\
i=1,2,3 .
\end{array}
$$

The directional Hubble parameters are obtained as $H_{i}=$ $\beta / 3+X_{0 i} / V_{0} e^{\beta t}, i=1,2,3$. The Hubble parameter $(H)$, deceleration parameter $(q)$, expansion scalar $(\Theta)$, and Shear scalar $\left(\sigma^{2}\right)$ are as follows:

$$
\begin{aligned}
H & =\frac{\beta}{3}, \\
q & =-1, \\
\Theta & =\beta, \\
\sigma^{2} & =\frac{X_{02}^{2}+X_{03}^{2}+X_{02} X_{03}}{V_{0}^{2} e^{2 \beta t}} .
\end{aligned}
$$

Here, we noticed that $\sigma^{2}$ die out for larger values of $t$. From (32) and (26), the energy density is expressed as

$$
\rho^{2}=\frac{\left(X_{02}^{2}+X_{03}^{2}+X_{02} X_{03}\right) e^{-2 \beta t}}{V_{0}^{2} \alpha(4 \pi+\lambda)}
$$




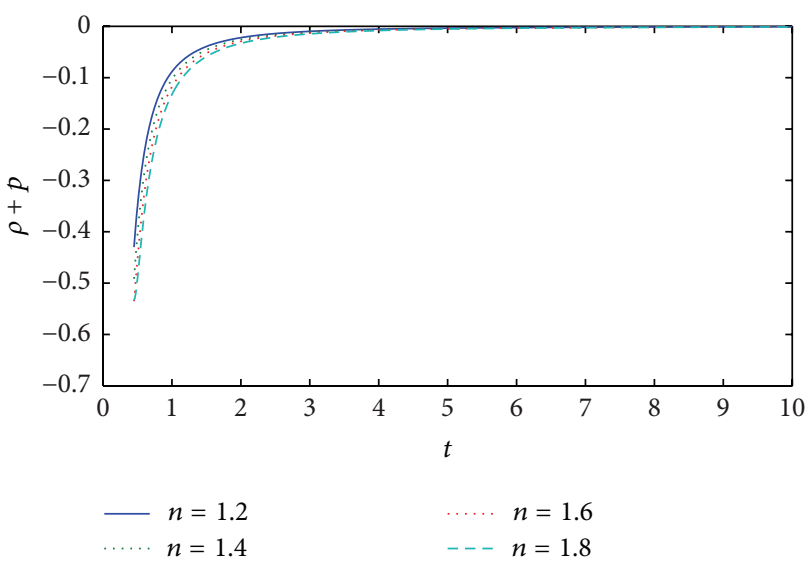

(a)

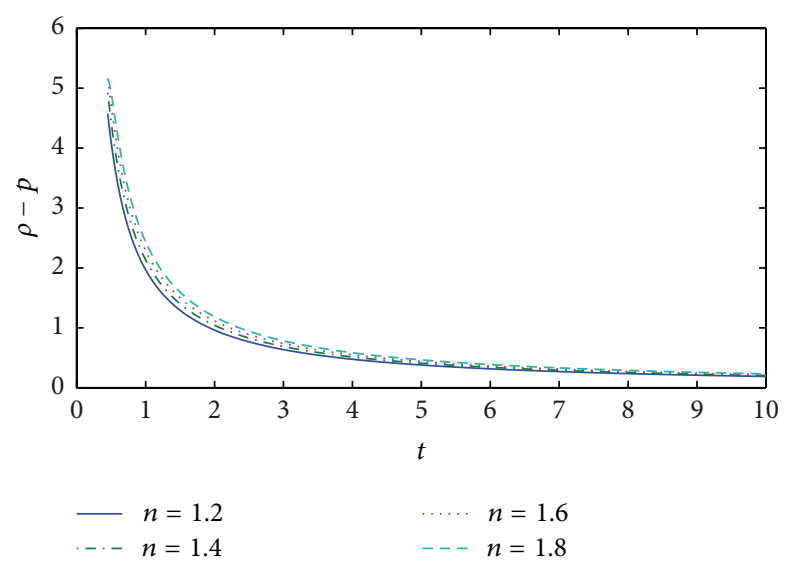

(b)

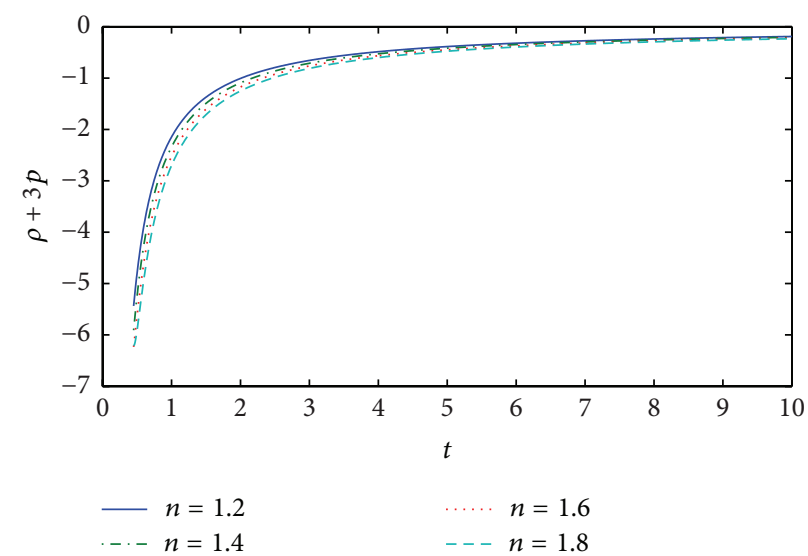

(c)

FIgURE 4: Variation of energy conditions $(\rho+p \geq 0, \rho-p \geq 0, \rho+3 p \geq 0)$ against time $t$ for power law model.

Using (34) in (3), the pressure is expressed as

$$
\begin{aligned}
p & =\frac{1}{(4 \pi+\lambda) V_{0}^{2}}[-(4 \pi+\lambda) \\
& \cdot V_{0}^{2} \sqrt{\frac{\left(X_{02}^{2}+X_{03}^{2}+X_{02} X_{03}\right) e^{-2 \beta t}}{V_{0}^{2} \alpha(4 \pi+\lambda)}} \\
& \left.+\left(X_{02}^{2}+X_{03}^{2}+X_{02} X_{03}\right) e^{-2 \beta t}\right] .
\end{aligned}
$$

With the help of (32)-(35) from (19), the cosmological constant $\Lambda$ is obtained as

$$
\begin{aligned}
\Lambda= & -(8 \pi+4 \lambda) \sqrt{\frac{\left(X_{02}^{2}+X_{03}^{2}+X_{02} X_{03}\right) e^{-2 \beta t}}{V_{0}^{2} \alpha(4 \pi+\lambda)}} \\
& +\frac{2(2 \pi+\lambda)\left(X_{02}^{2}+X_{03}^{2}+X_{02} X_{03}\right)}{e^{2 \beta t} V_{0}^{2}(4 \pi+\lambda)}-\frac{\beta^{2}}{3} .
\end{aligned}
$$

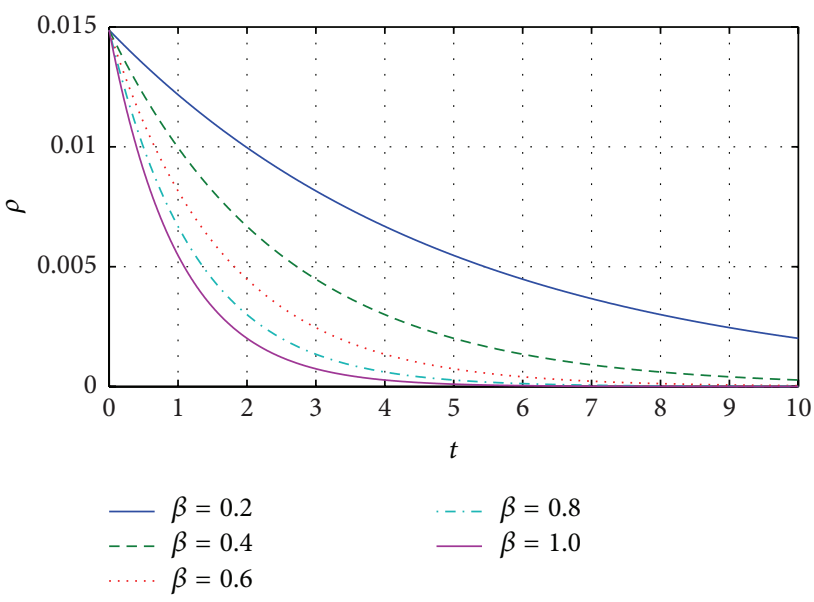

FIgURE 5: Variation of energy density $\rho$ against time $t$ for $\lambda=$ $1, \alpha=0.1, V_{0}=1, X_{02}=0.01, X_{03}=0.01$, and different $\beta(0.2,0.4,0.6,0.8,1)$.

Figures 5 and 7 show the variation of energy density $\rho$ and pressure $p$ against time $t$ for different values as in the figures. Here, we noticed that $\rho, p \rightarrow 0$ when $t \rightarrow \infty$. 


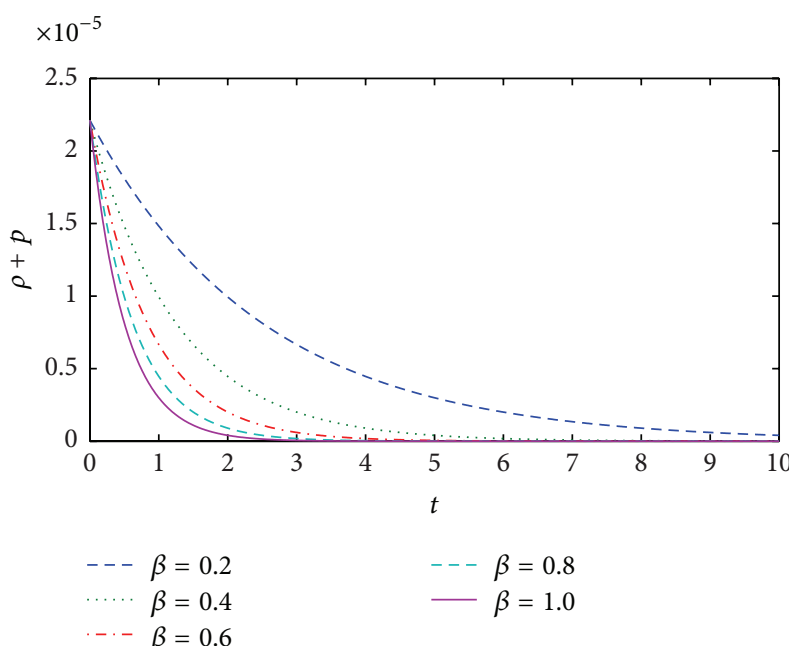

(a)

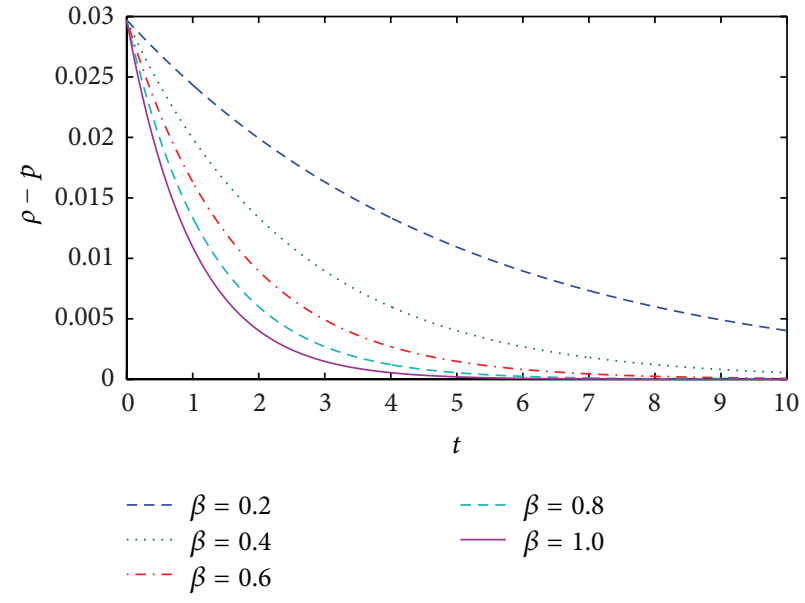

(b)

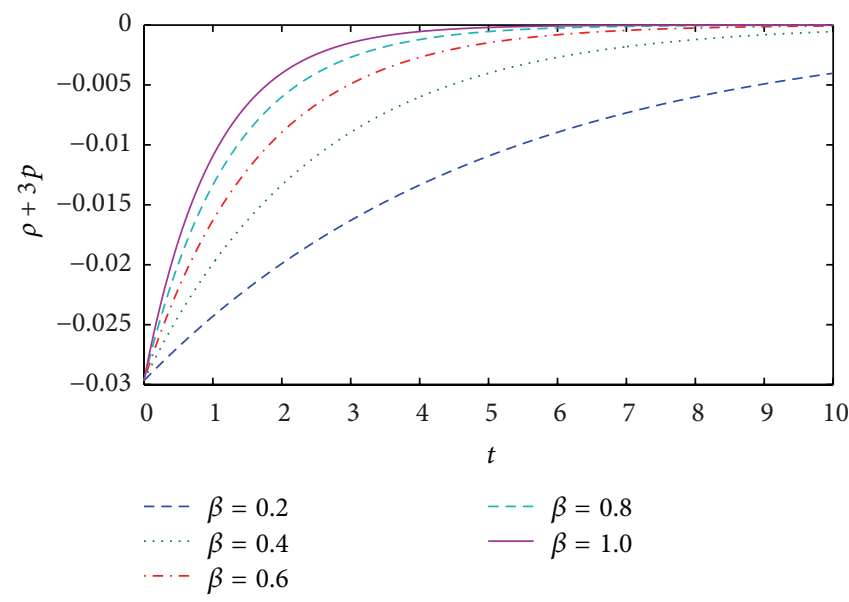

(c)

FIgURE 6: Variation of energy conditions $(\rho+p \geq 0, \rho-p \geq 0, \rho+3 p \geq 0)$ against time $t$ for exponential law model.

In the increase of $\beta$, energy density and pressure decrease, respectively. Variation of different energy conditions against time for different $n$ is presented in Figure 6. We observed from the figure that NEC and DEC are satisfied, but in this case SEC is violated. This violation may be responsible for the accelerated expansion of the universe. Figure 8 represents the variation of cosmological constant $\Lambda$ against time $t$ for different values as in the figures. It is observed that cosmological constant $\Lambda$ is not approaching towards zero with the evolution of time and also it takes negative values.

\section{Field Equations and Cosmological Model for}

$$
f(R, T)=f_{1}(R)+f_{2}(T)
$$

In $f(R, T)$ theory, the gravitational field equation (15) for the choice of $f_{1}(R)=\lambda R$ and $f_{2}(T)=\lambda T$, along with cosmological constant $\Lambda$, is given as

$$
G_{i j}=\left(\frac{8 \pi+\lambda}{\lambda}\right) T_{i j}+\left(\frac{\rho-p+2 \Lambda}{2}\right) g_{i j} .
$$

In this case, the field equations are given by

$$
\begin{gathered}
\frac{\dot{X}_{1} \dot{X}_{2}}{X_{1} X_{2}}+\frac{\dot{X}_{1} \dot{X}_{3}}{X_{1} X_{3}}+\frac{\dot{X}_{2} \dot{X}_{3}}{X_{2} X_{3}}=-\left(\frac{16 \pi+3 \lambda}{2 \lambda}\right) \rho+\frac{p}{2}-\Lambda \\
\frac{\ddot{X}_{2}}{X_{2}}+\frac{\ddot{X}_{3}}{X_{3}}+\frac{\dot{X}_{2} \dot{X}_{3}}{X_{2} X_{3}}=\left(\frac{16 \pi+3 \lambda}{2 \lambda}\right) p-\frac{\rho}{2}-\Lambda \\
\frac{\ddot{X}_{1}}{X_{1}}+\frac{\ddot{X}_{3}}{X_{3}}+\frac{\dot{X}_{1} \dot{X}_{3}}{X_{1} X_{3}}=\left(\frac{16 \pi+3 \lambda}{2 \lambda}\right) p-\frac{\rho}{2}-\Lambda \\
\frac{\ddot{X}_{1}}{X_{1}}+\frac{\ddot{X}_{2}}{X_{2}}+\frac{\dot{X}_{1} \dot{X}_{2}}{X_{1} X_{2}}=\left(\frac{16 \pi+3 \lambda}{2 \lambda}\right) p-\frac{\rho}{2}-\Lambda .
\end{gathered}
$$

5.1. Power Law Model. Following the same procedure as in Section 4.1, we have obtained the same metric potential as in 


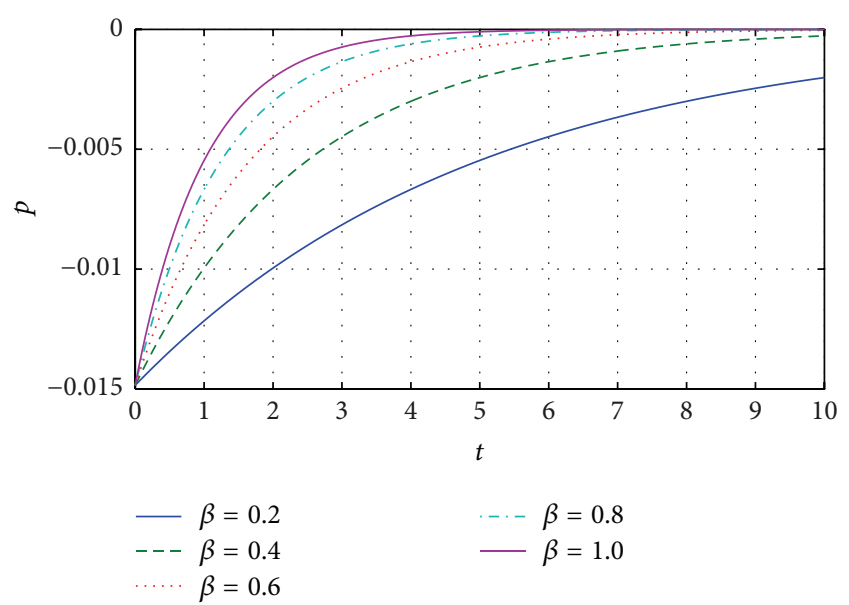

FIGURE 7: Variation of pressure $p$ against time $t$ for $\lambda=1, \alpha=0.1$, $V_{0}=1, X_{02}=0.01, X_{03}=0.01$, and different $\beta(0.2,0.4,0.6,0.8,1)$.

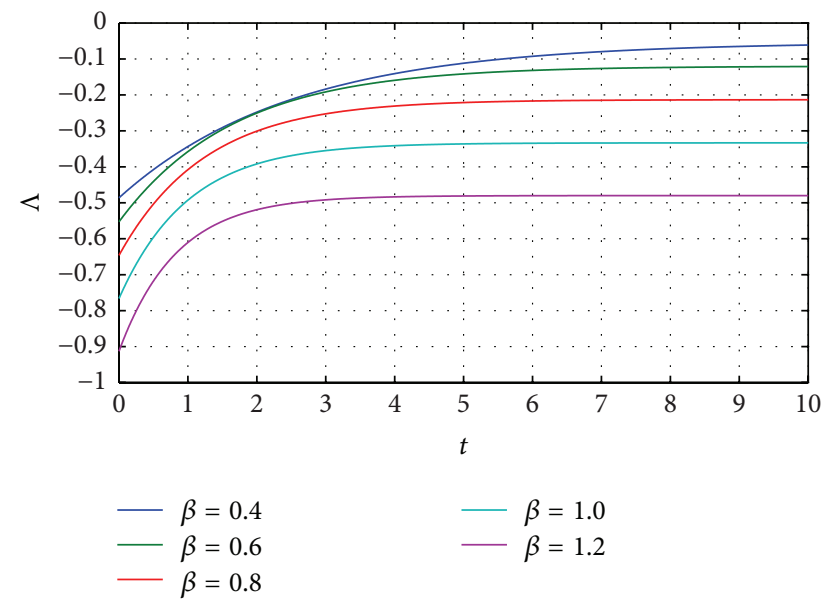

Figure 8: Variation of cosmological constant $\Lambda$ against time $t$ for $\lambda=1, \alpha=0.1, V_{0}=1, X_{02}=0.01, X_{03}=0.01$, and different $\beta(0.4,0.6,0.8,1,1.2)$.

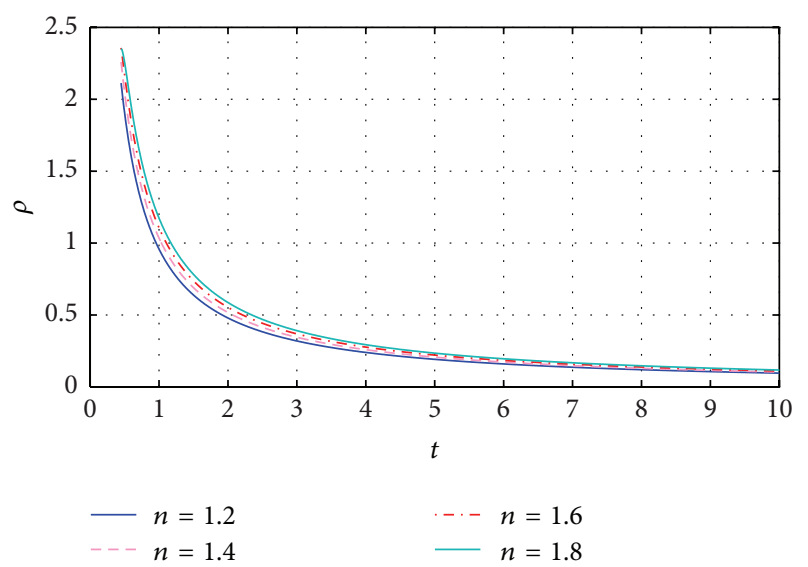

FIGURE 9: Variation of energy density $\rho$ against time $t$ for $\lambda=1$, $\alpha=-0.1, V_{0}=1, X_{02}=0.01, X_{03}=0.01$, and different $n(1.2,1.4,1.6,1.8)$.

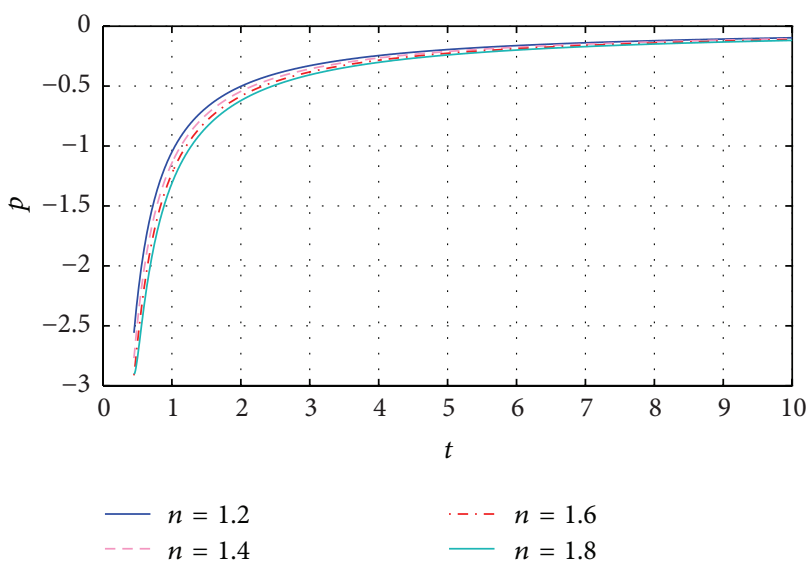

FIgURE 10: Variation of pressure $p$ against time $t$ for $\lambda=1, \alpha=-0.1$, $V_{0}=1, X_{02}=0.01, X_{03}=0.01$, and different $n(1.2,1.4,1.6,1.8)$.

(27) and the other parameters like energy density $\rho$, pressure $p$, and cosmological constant $\Lambda$ are expressed as follows:

$$
\begin{aligned}
\rho^{2} & =\frac{2 \lambda}{V_{0}^{2} \alpha(8 \pi+\lambda)}\left[\frac{X_{03}^{2}+X_{02}^{2}+X_{02} X_{03}}{t^{6 n}}-\frac{V_{0}^{2} n}{t^{2}}\right] \\
p & =\frac{2 \lambda}{V_{0}^{2}(8 \pi+\lambda)}\left[\frac{X_{03}^{2}+X_{02}^{2}+X_{02} X_{03}}{t^{6 n}}-\frac{V_{0}^{2} n}{t^{2}}\right] \\
& -\sqrt{\frac{2 \lambda}{V_{0}^{2} \alpha(8 \pi+\lambda)}\left[\frac{X_{03}^{2}+X_{02}^{2}+X_{02} X_{03}}{t^{6 n}}-\frac{V_{0}^{2} n}{t^{2}}\right]} \\
\Lambda & =\frac{1}{2 t^{2} \lambda(8 \pi+\lambda) \alpha V_{0}^{2}}[-41 \sqrt{\lambda}(4 \pi+\lambda) \\
& \cdot \sqrt{8 \pi+\lambda \sqrt{\alpha} V_{0} t^{-3 n+1} \sqrt{2 V_{0}^{2} n t^{6 n}-2 t^{2}\left(X_{03}^{2}+X_{02}^{2}+X_{02} X_{03}\right)}} \\
& +4(4 \pi+\lambda)\left(X_{03}^{2}+X_{02}^{2}+X_{02} X_{03}\right) \lambda \alpha t^{-6 n+2} \\
& \left.-2 n(3 \lambda n+\lambda+24 n \pi) V_{0}^{2} \alpha \lambda\right] .
\end{aligned}
$$

Here, also we have noticed similar qualitative results as in Section 4.1 (see Figures 9-12).

5.2. Exponential Law Model. Following the same procedure as in Section 4.2, we have obtained the same metric potential as in (34), and the other parameters like energy density $\rho$, pressure $p$, and cosmological constant $\Lambda$ are as follows:

$$
\rho=\frac{1}{V_{0} e^{\beta t}} \sqrt{\frac{2\left(X_{03}^{2}+X_{02}^{2}+X_{02} X_{03}\right) \lambda}{\alpha(8 \pi+\lambda)}}
$$




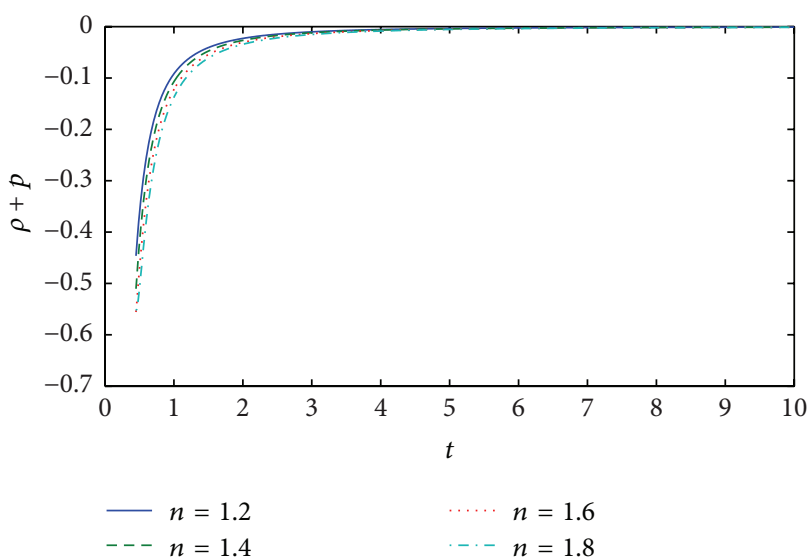

(a)
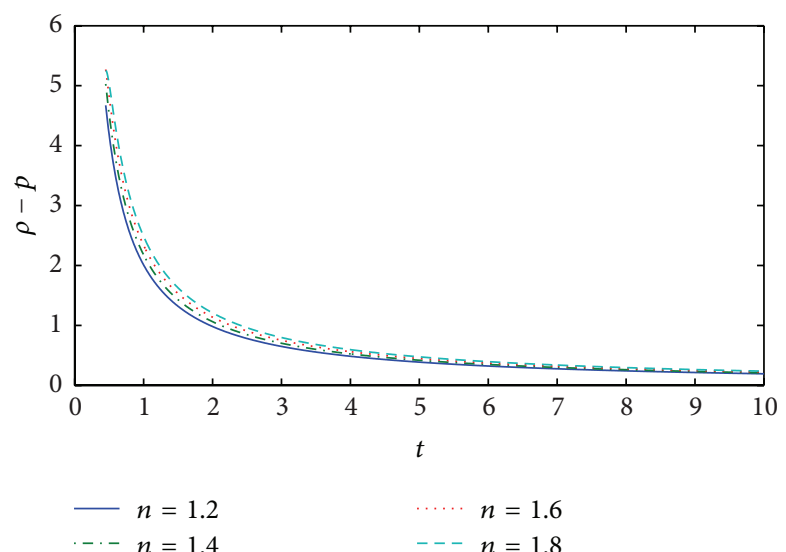

$n=1.6$

(b)

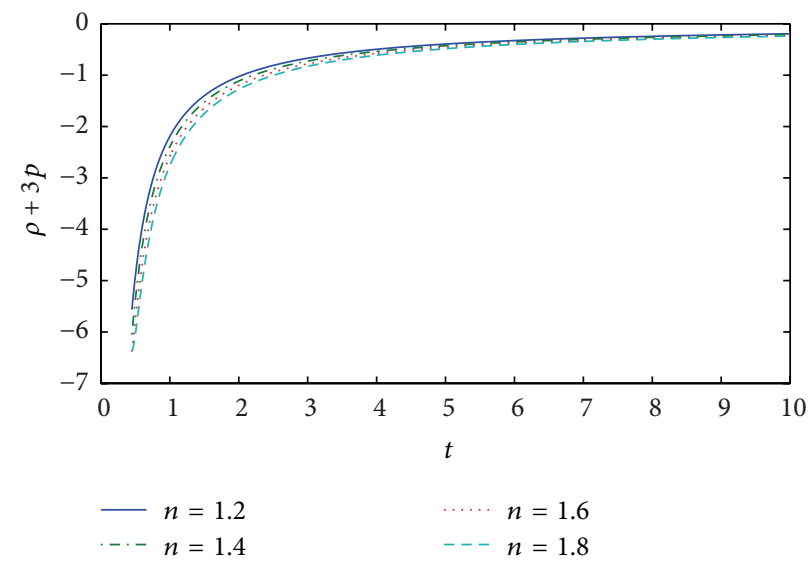

(c)

FIGURE 11: Variation of energy conditions $(\rho+p \geq 0, \rho-p \geq 0, \rho+3 p \geq 0)$ against time $t$ for polynomial law model.

$p$

$$
\begin{aligned}
= & \frac{2\left(X_{03}^{2}+X_{02}^{2}+X_{02} X_{03}\right) \lambda}{V_{0}^{2} e^{2 \beta t}(8 \pi+\lambda)} \\
& -\frac{1}{V_{0} e^{\beta t}} \sqrt{\frac{2\left(X_{03}^{2}+X_{02}^{2}+X_{02} X_{03}\right) \lambda}{\alpha(8 \pi+\lambda)}}
\end{aligned}
$$

$\Lambda$

$$
\begin{gathered}
=-\frac{2 \sqrt{2}(4 \pi+\lambda)}{\lambda} \sqrt{\frac{2\left(X_{03}^{2}+X_{02}^{2}+X_{02} X_{03}\right) \lambda e^{-2 \beta t}}{V_{0}^{2} \alpha(8 \pi+\lambda)}} \\
+\frac{2\left(X_{03}^{2}+X_{02}^{2}+X_{02} X_{03}\right)(4 \pi+\lambda) e^{-2 \beta t}}{V_{0}^{2}(8 \pi+\lambda)}-\frac{\beta^{2}}{3} .
\end{gathered}
$$

Here, also we have noticed the similar qualitative results as in Section 4.2 (see Figures 13-16).

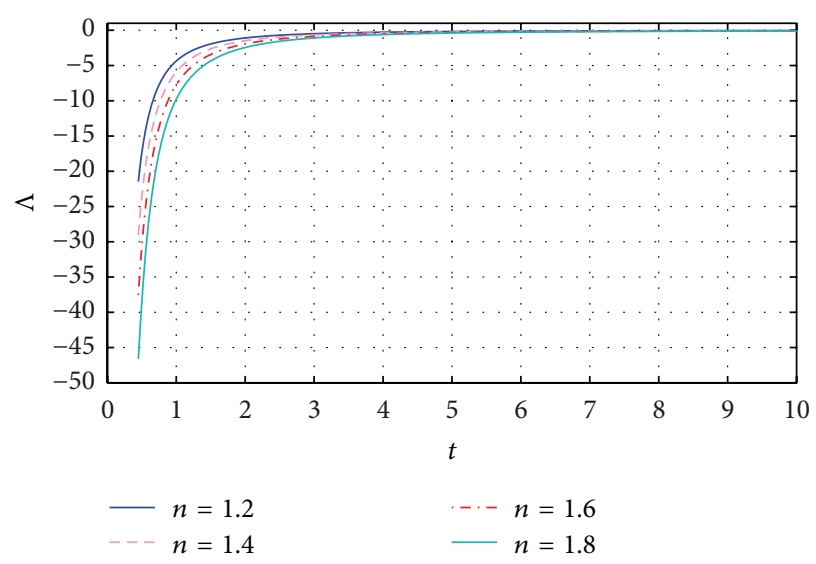

FIGURE 12: Variation of cosmological constant $\Lambda$ against time $t$ for $\lambda=1, \alpha=-0.1, V_{0}=1, X_{02}=0.01, X_{03}=0.01$, and different $n(1.2,1.4,1.6,1.8)$.

\section{Concluding Remarks}

In this paper, we have the Bianchi type-I cosmological model in $f(R, T)$ modified gravity for two different classes of $f(R, T)$ 


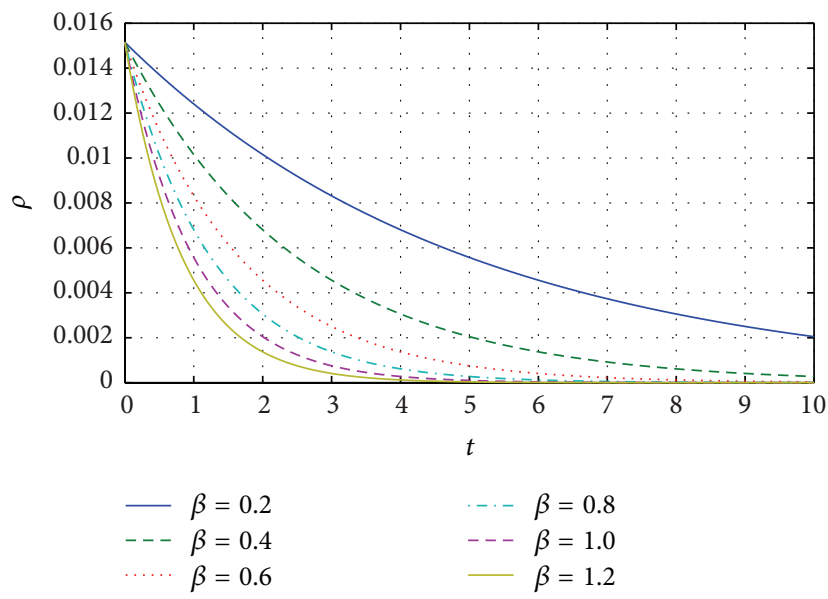

Figure 13: Variation of energy density $\rho$ against time $t$ for $\lambda=$ $1, \alpha=0.1, V_{0}=1, X_{02}=0.01, X_{03}=0.01$, and different $\beta(0.2,0.4,0.6,0.8,1,1.2)$.

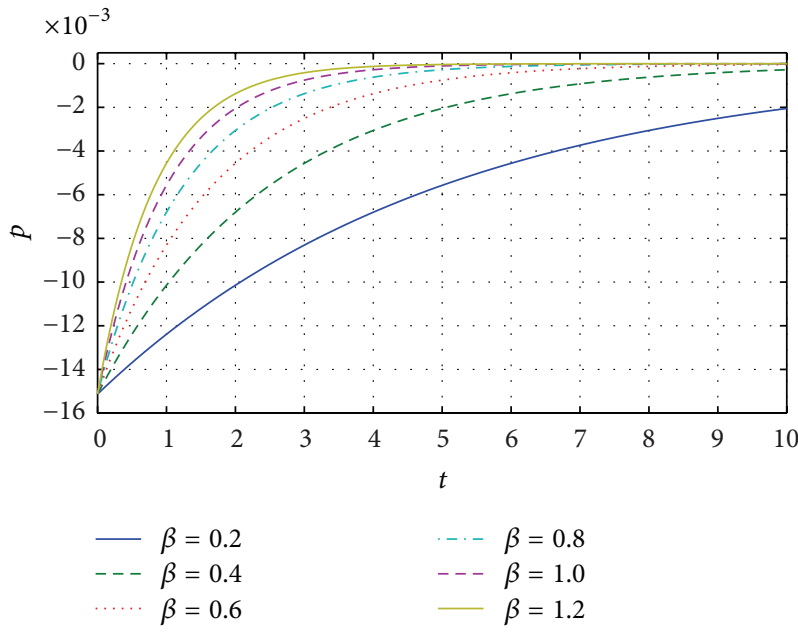

FIgURE 14: Variation of pressure $p$ against time $t$ for $\lambda=1$, $\alpha=0.1, V_{0}=1, X_{02}=0.01, X_{03}=0.01$, and different $\beta(0.2,0.4,0.6,0.8,1,1.2)$.

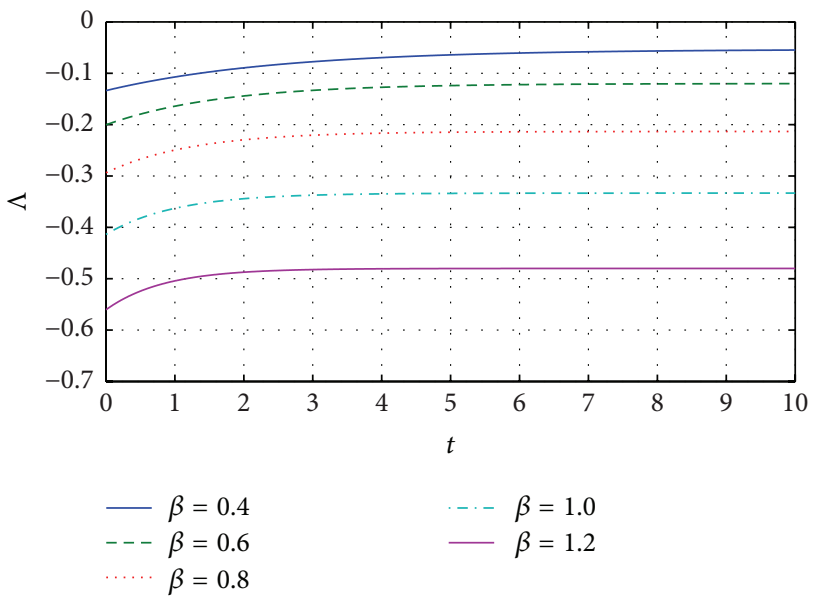

FIgURE 15: Variation of cosmological constant $\Lambda$ against time $t$ for $\lambda=1, \alpha=0.1, V_{0}=1, X_{02}=0.01, X_{03}=0.01$, and different $\beta(0.4,0.6,0.8,1,1.2)$.

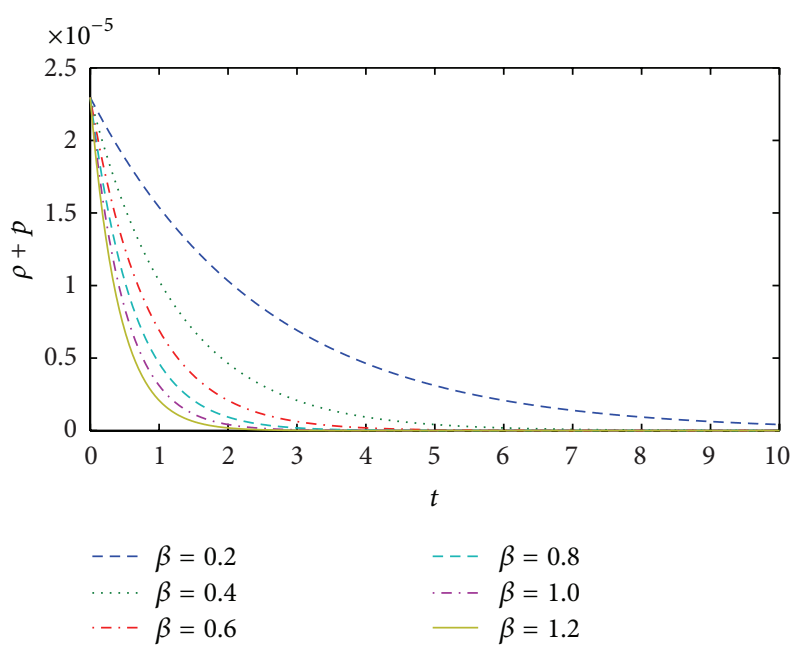

(a)

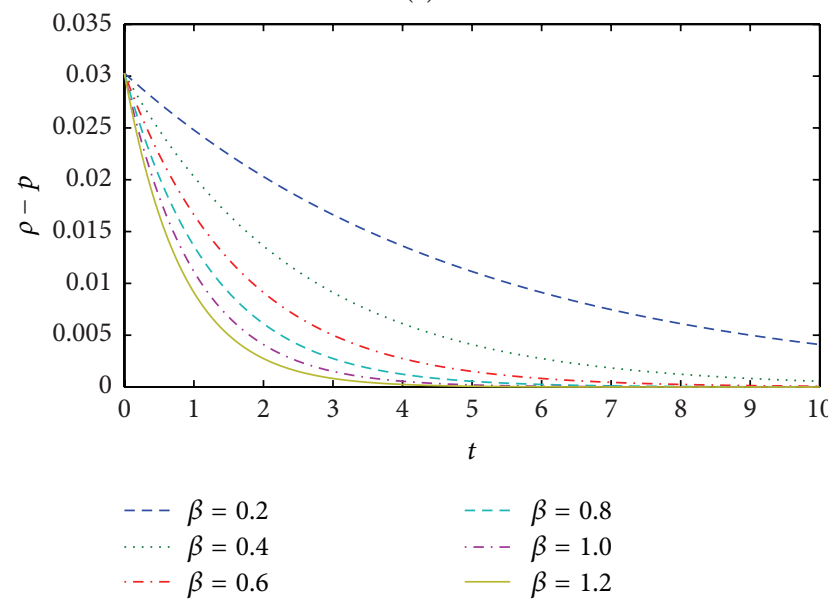

(b)

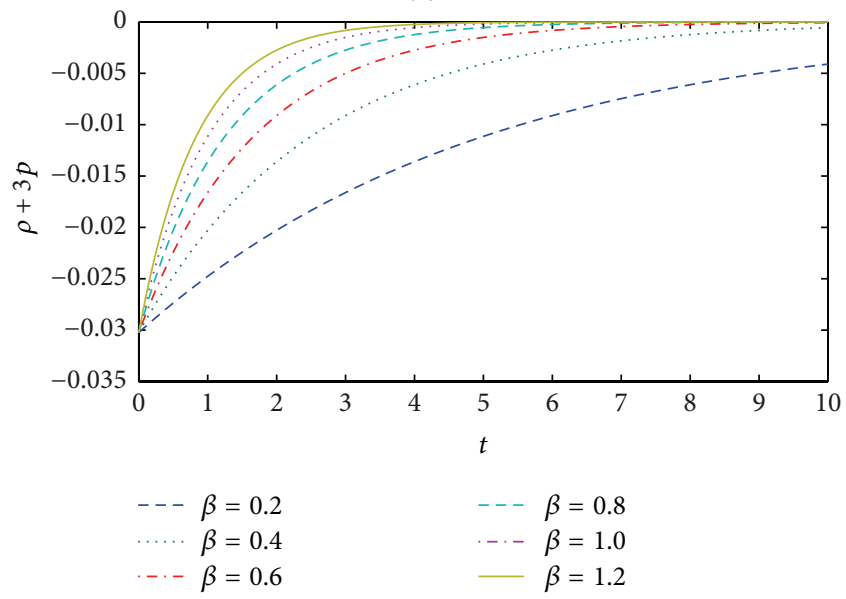

(c)

FIGURE 16: Variation of energy conditions $(\rho+p \geq 0, \rho-p \geq 0$, $\rho+3 p \geq 0$ ) against time $t$ for polynomial law model.

in the presence of cosmological constant and quadratic equation of state. Here, we have discussed two models based on the expansion law. From both models, case of $f(R, T)=$ $R+2 f(T)$, we have concluded the following points. 
(i) In both the models, energy density $\rho$ is decreasing function of $t$ and $\rho$ approaches towards zero with the evolution of time.

(ii) In both the models, pressure $p$ is negative and approaches towards zero with the evolution of time.

(iii) In both the models, cosmological constant $\Lambda$ is negative, but here we notice that in case of power law $\Lambda$ approaches towards zero with the evolution of time whereas it does not approach towards zero with the evolution of time in case of exponential law.

Similar qualitative observations are also noticed for the case of $f(R, T)=f_{1}(R)+f_{2}(T)$. Here, all the observation are in fare agreement with the observational data.

\section{Conflict of Interests}

The authors declare that there is no conflict of interests regarding the publication of this paper.

\section{References}

[1] A. G. Riess, A. V. Filippenko, P. Challis et al., "Observational evidence from supernovae for an accelerating universe and a cosmological constant," The Astronomical Journal, vol. 116, no. 3, pp. 1009-1038, 1998.

[2] S. Perlmutter, G. Aldering, G. Goldhaber et al., "Measurements of $\Omega$ and $\Lambda$ from 42 high-redshift supernovae," The Astrophysical Journal, vol. 517, no. 2, pp. 565-586, 1999.

[3] W. J. Percival, "The build-up of haloes within press-schechter theory," Monthly Notices of the Royal Astronomical Society, vol. 327, no. 4, pp. 1313-1322, 2001.

[4] R. Jimenez, L. Verde, T. Treu, and D. Stern, "Constraints on the equation of state of dark energy and the Hubble constant from stellar ages and the cosmic microwave background," The Astrophysical Journal, vol. 593, no. 2, pp. 622-629, 2003.

[5] D. Stern, R. Jimenez, L. Verde, M. K. Kowski, and S. A. Stanford, "Cosmic chronometers: constraining the equation of state of dark energy. I: $H(z)$ measurements," Journal of Cosmology and Astroparticle Physics, vol. 2010, no. 2, article 008, 2010.

[6] P. J. E. Peebles and B. Ratra, "The cosmological constant and dark energy," Reviews of Modern Physics, vol. 75, no. 2, pp. 559606, 2003.

[7] V. Sahni and A. A. Starobinsky, "The case for a positive cosmological $\Lambda$-term," International Journal of Modern Physics D, vol. 9, no. 4, pp. 373-444, 2000.

[8] J. Martin, "Quintessence: a mini-review," Modern Physics Letters A, vol. 23, no. 17-20, pp. 1252-1265, 2008.

[9] S. Nojiri, S. D. Odintsov, and M. Sami, "Dark energy cosmology from higher-order, string-inspired gravity, and its reconstruction," Physical Review D: Particles, Fields, Gravitation and Cosmology, vol. 74, no. 4, Article ID 046004, 2006.

[10] T. Chiba, T. Okabe, and M. Yamaguchi, "Kinetically driven quintessence," Physical Review D, vol. 62, no. 2, Article ID 023511, 2000.

[11] T. Padmanabhan and T. R. Choudhury, "Can the clustered dark matter and the smooth dark energy arise from the same scalar field?" Physical Review D, vol. 66, no. 8, Article ID 081301, pp. 813011-813014, 2002.
[12] M. C. Bento, O. Bertolami, and A. A. Sen, "Generalized Chaplygin gas, accelerated expansion, and dark-energy-matter unification," Physical Review D, vol. 66, no. 4, Article ID 043507, 2002.

[13] L. O. Pimentel and L. M. Diaz-Rivera, "Coasting cosmologies with time dependent cosmological constant," International Journal of Modern Physics A, vol. 14, no. 10, pp. 1523-1529, 1999.

[14] G. P. Singh, S. Kotambar, and A. Pradhan, "Cosmological models with a variable $\Lambda$ term in higher dimensional spacetime," Fizika B, vol. 15, p. 23, 2006.

[15] G. P. Singh, A. Y. Kale, and J. Tripathi, "Dynamic cosmological 'constant' in brans dicke theory," Romanian Journal of Physics, vol. 58, no. 1-2, pp. 23-35, 2013.

[16] M. Jamil and U. Debnath, "FRW cosmology with variable $G$ and $\Lambda$," International Journal of Theoretical Physics, vol. 50, no. 5, pp. 1602-1613, 2011.

[17] G. Magnano, M. Ferraris, and M. Francaviglia, "Nonlinear gravitational Lagrangians," General Relativity and Gravitation, vol. 19, no. 5, pp. 465-479, 1987.

[18] M. Sharif and S. Azeem, "Cosmological evolution for dark energy models in $f(T)$ gravity," Astrophysics and Space Science, vol. 342, no. 2, pp. 521-530, 2012.

[19] M. Jamil, D. Momeni, and R. Myrzakulov, "Attractor solutions in $f(T)$ cosmology," The European Physical Journal C, vol. 72, no. 3, pp. 1-10, 2012.

[20] K. Bamba, M. Jamil, D. Momeni, and R. Myrzakulov, "Generalized second law of thermodynamics in $f(T)$ gravity with entropy corrections," Astrophysics and Space Science, vol. 344, no. 1, pp. 259-267, 2013.

[21] A. de Felice and S. Tsujikawa, " $f(R)$ theories," Living Reviews in Relativity, vol. 13, no. 3, pp. 1-161, 2010.

[22] T. P. Sotiriou and V. Faraoni, " $f(T)$ theories of gravity," Reviews of Modern Physics, vol. 82, no. 1, pp. 451-497, 2010.

[23] S. Nojiri and S. D. Odintsov, "Unified cosmic history in modified gravity: from $F(R)$ theory to Lorentz non-invariant models," Physics Reports, vol. 505, no. 2-4, pp. 59-144, 2011.

[24] L. Amendola, R. Gannouji, D. Polarski, and S. Tsujikawa, "Conditions for the cosmological viability of $f(R)$ dark energy models," Physical Review D, vol. 75, no. 8, Article ID 083504, 2007.

[25] M. Jamil, F. M. Mahomed, and D. Momeni, "Noether symmetry approach in $f(R)$-tachyon model," Physics Letters, Section B: Nuclear, Elementary Particle and High-Energy Physics, vol. 702, no. 5, pp. 315-319, 2011.

[26] A. Azadi, D. Momeni, and M. Nouri-Zonoz, "Cylindrical solutions in metric $f(R)$ gravity," Physics Letters B, vol. 670, no. 3, pp. 210-214, 2008.

[27] D. Momeni and H. Gholizade, "A note on constant curvature solutions in cylindrically symmetric metric $f(R)$ gravity," International Journal of Modern Physics D, vol. 18, no. 11, pp. 17191729, 2009.

[28] T. Harko, F. S. N. Lobo, S. Nojiri, and S. D. Odintsov, " $f(R$, T) gravity," Physical Review D: Particles, Fields, Gravitation and Cosmology, vol. 84, no. 2, Article ID 024020, 2011.

[29] J. Barrientos and G. F. Rubilar, "Comment on ' $f(R, T)$ gravity", Physical Review D, vol. 90, no. 2, Article ID 028501, 2014.

[30] M. Jamil, D. Momeni, M. Raza, and R. Myrzakulov, "Reconstruction of some cosmological models in $f(R, T)$ cosmology," The European Physical Journal C, vol. 72, article 1999, 2012.

[31] M. Jamil, D. Momeni, and R. Myrzakulov, "Violation of the first law of thermodynamics in $f(R, T)$ gravity," Chinese Physics Letters, vol. 29, no. 10, Article ID 109801, 2012. 
[32] D. Momeni, R. Myrzakulov, and E. Güdekli, "Cosmological viable Mimetic $f(R)$ and $f(R, T)$ theories via Noether symmetry," International Journal of Geometric Methods in Modern Physics, vol. 12, no. 10, Article ID 1550101, 2015.

[33] M. Jamil, S. Ali, D. Momeni, and R. Myrzakulov, "Bianchi type I cosmology in generalized Saez-Ballester theory via Noether gauge symmetry," The European Physical Journal C, vol. 72, no. 4, pp. 1-6, 2012.

[34] M. Jamil, D. Momeni, N. S. Serikbayev, and R. Myrzakulov, "FRW and Bianchi type I cosmology of f-essence," Astrophysics and Space Science, vol. 339, no. 1, pp. 37-43, 2012.

[35] P. K. Sahoo and M. Sivakumar, "LRS Bianchi type-I cosmological model in $f(R, T)$ theory of gravity with $\Lambda(T)$," Astrophysics and Space Science, vol. 357, no. 1, article 60, 2015.

[36] N. Ahmed and A. Pradhan, "Bianchi type-V cosmology in $f(R ; T)$ gravity with $\Lambda(T)$," International Journal of Theoretical Physics, vol. 53, no. 1, pp. 289-306, 2014.

[37] A. Pradhan, N. Ahmed, and B. Saha, "Reconstruction of modified $f(R, T)$ with $\Lambda(T)$ gravity in general class of Bianchi cosmological models," Canadian Journal of Physics, vol. 93, no. 6, pp. 654-662, 2015.

[38] S. Nojiri and S. D. Odintsov, "Final state and thermodynamics of a dark energy universe," Physical Review D: Particles, Fields, Gravitation and Cosmology, vol. 70, no. 10, Article ID 103522, 2004.

[39] K. N. Ananda and M. Bruni, "Cosmological dynamics and dark energy with a nonlinear equation of state: a quadratic model," Physical Review D, vol. 74, no. 2, Article ID 023523, 2006.

[40] A. V. Astashenok, S. Nojiri, S. D. Odintsov, and R. J. Scherrer, "Scalar dark energy models mimicking $\Lambda$ CDM with arbitrary future evolution," Physics Letters B, vol. 713, no. 3, pp. 145-153, 2012.

[41] S. Nojiri and S. D. Odintsov, "Inhomogeneous equation of state of the universe: phantom era, future singularity, and crossing the phantom barrier," Physical Review D: Particles, Fields, Gravitation and Cosmology, vol. 72, no. 2, Article ID $023003,2005$.

[42] S. Capozziello, V. F. Cardone, E. Elizalde, S. Nojiri, and S. D. Odintsov, "Observational constraints on dark energy with generalized equations of state," Physical Review D, vol. 73, no. 4, Article ID 043512, 2006.

[43] F. Rahaman, M. Jamil, and K. Chakraborty, "Revisiting the classical electron model in general relativity," Astrophysics and Space Science, vol. 331, no. 1, pp. 191-197, 2010.

[44] T. Feroze and A. A. Siddiqui, "Charged anisotropic matter with quadratic equation of state," General Relativity and Gravitation, vol. 43, no. 4, pp. 1025-1035, 2011.

[45] S. D. Maharaj and P. Mafa Takisa, "Regular models with quadratic equation of state," General Relativity and Gravitation, vol. 44, no. 6, pp. 1419-1432, 2012.

[46] P. H. Chavanis, "A cosmological model based on a quadratic equation of state unifying vacuum energy, radiation, and dark energy," Journal of Gravity, vol. 2013, Article ID 682451, 20 pages, 2013.

[47] P.-H. Chavanis, "A cosmological model describing the early inflation, the intermediate decelerating expansion, and the late accelerating expansion by a quadratic equationof state," http://arxiv.org/abs/1309.5784.

[48] M. Malaver, "Strange quark star model with quadratic equation of state," Frontiers of Mathematics and Its Applications, vol. 1, no. 1, pp. 9-15, 2014.
[49] D. R. K. Reddy, K. S. Adhav, and M. A. Purandare, "Bianchi type-I cosmological model with quadratic equation of state," Astrophysics and Space Science, vol. 357, no. 1, article 20, 2015.

[50] S. Kotambkar, G. P. Singh, and R. Kelkar, "Anisotropic cosmological models with quintessence," International Journal of Theoretical Physics, vol. 53, no. 2, pp. 449-460, 2014. 

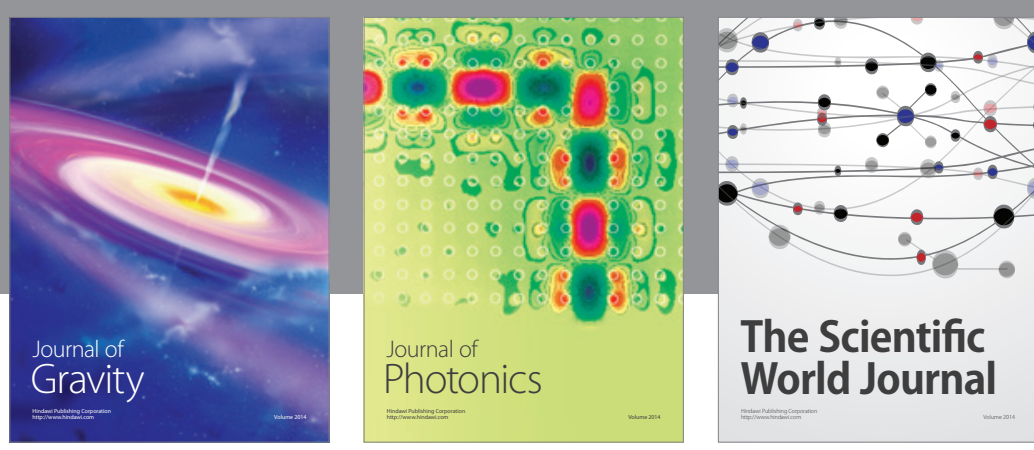

The Scientific World Journal
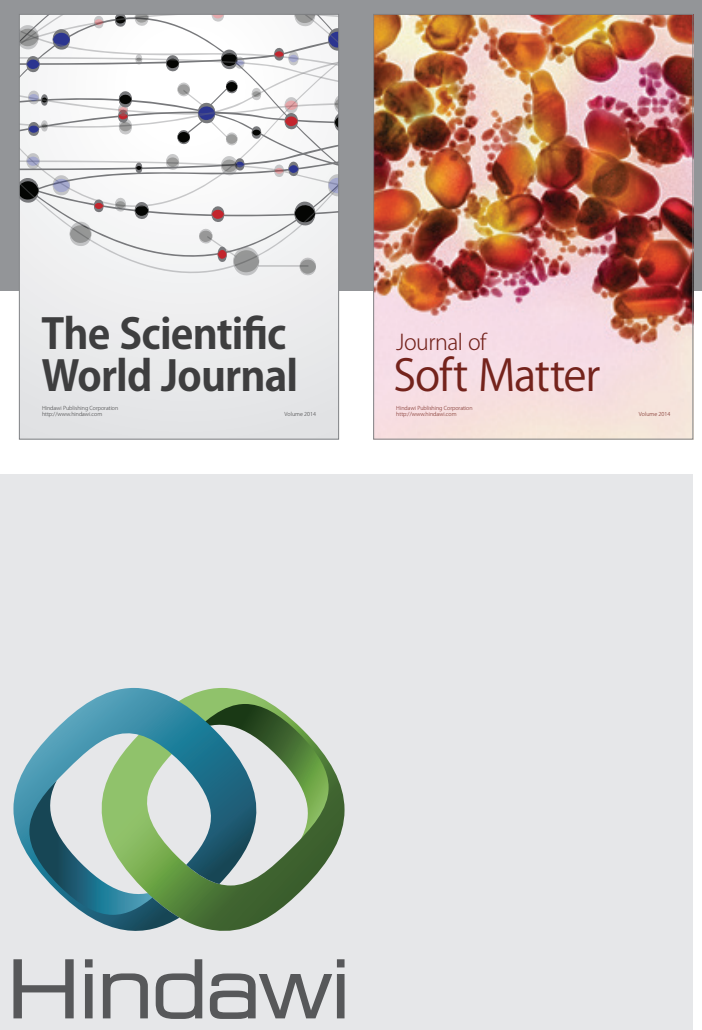

Submit your manuscripts at

http://www.hindawi.com

nternational Journal of

Statistical Mechanics
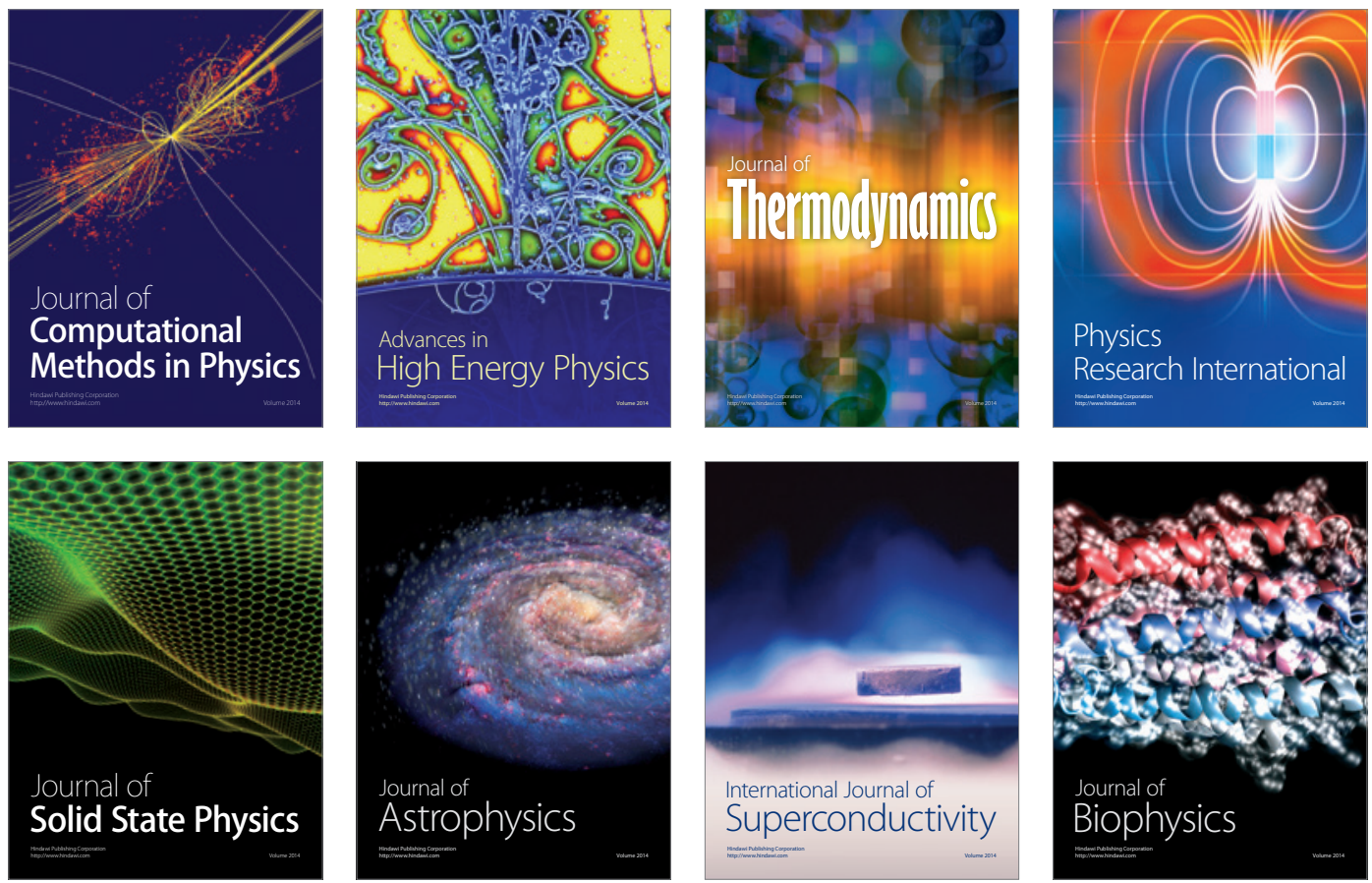
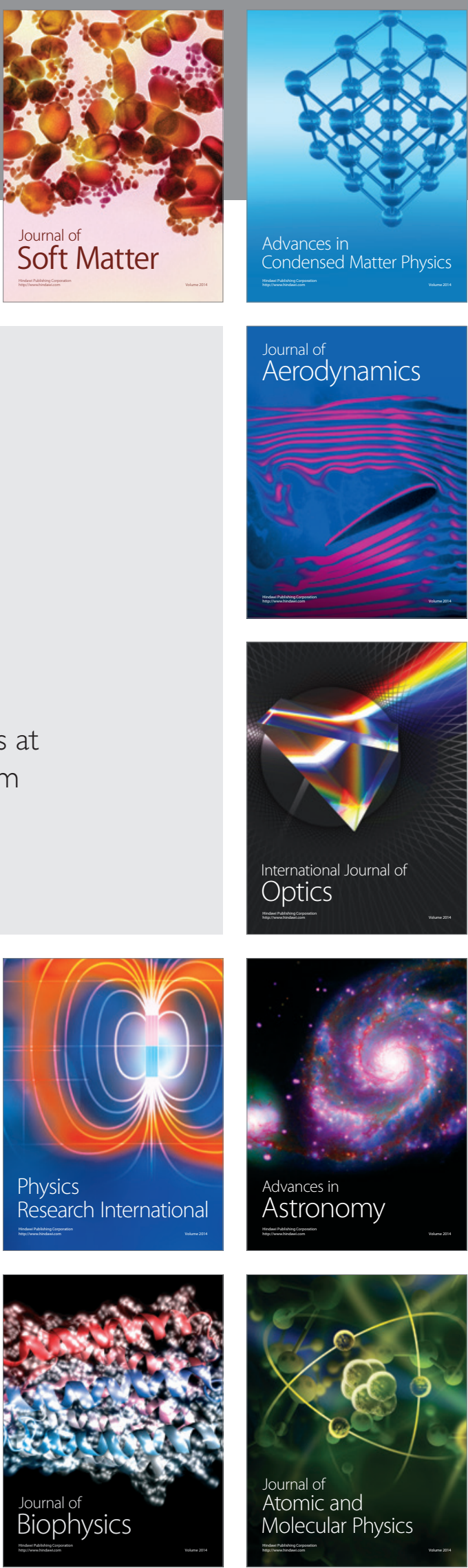